\title{
The Safety and Efficiency of Parent Artery Occlusion according to the Location of Occlusion
}

\section{Wong $\mathrm{H}^{1}$, Botsford $\mathrm{A}^{1}$ and Shankar $\mathrm{J}^{1,2 *}$}

${ }^{1}$ Department of Diagnostic Radiology, Dalhousie University, Canada

${ }^{2}$ Department of Radiology, University of Manitoba, Canada

*Corresponding author: Jai Shankar, Department of Diagnostic Radiology, Dalhousie University, Halifax, Nova Scotia \& Department of Radiology, University of Manitoba, Winnipeg, Manitoba, 1 Evengreen Place, Winnipeg, MB, R3L 0E9, Canada, Tel: 431-373-4164; Email: shivajai1@gmail.com

\section{Research Article}

Volume 4 Issue 1

Received Date: January 27, 2020

Published Date: March 03, 2020

DOI: $10.23880 /$ crij-16000168

\section{Abstract}

Background and Purpose: Reconstructive treatment methods for aneurysms are increasing, but their use over traditional endovascular parent artery occlusion (PAO) remains debated. The purpose of this study was to examine the safety and efficacy of planned intra- and extracranial PAO.

Materials and Methods: A retrospective chart review was conducted on consecutive patients who underwent endovascular treatment for PAO at our institution between 1999 and 2017. Patients who underwent planned and intentional PAO were included in the study. Information was collected on demographics, clinical symptoms, imaging findings, and follow-up outcomes. Findings were presented according to PAO performed intra- and extra-cranially.

Results: Among 1000 endovascular treatment, 32 patients (19 women; 13 men; mean age 53 \pm 13.2 years) had planned PAO. PAO was performed intracranially in 11 and extracranially in 21 patients. Permanent neurological deficits were seen in $36.36 \%$ and $4.76 \%$ of intracranial and extracranial PAO, respectively. Intracranial PAO was independently associated with higher neurological complications than extracranial PAO (odds ratio: 11.43; 95\% confidence interval: 0.83-158.07; p-value: 0.02). No patients with PAO with balloon test occlusion (BTO) had any perioperative neurological complications.

Conclusion: Efficacy of PAO in our small study was 100\%. Intracranial PAO was an independent predictor for associated neurological morbidity compared to extracranial PAO. BTO appeared to help avoid neurological complications.

Keywords: Extracranial; Aneurysms; Vertebral arteries; Thrombosed; Cerebral circulation; Embolism

Abbreviations: PAO: Parent Artery Occlusion; BTO: Balloon Test Occlusion; ICA: Internal Carotid Artery; ACA: Anterior Cerebral Artery; MCA: Middle Cerebral Artery; PCA: Posterior Cerebral Artery; OR: Odds Ratio; CoW: Circle of Willis; CTA: Computed Tomography Angiography; DSA: Digital Subtraction Angiography; CT: Computed Tomography; DSA: Digital Subtraction Angiography.

\section{Introduction}

Management of intracranial aneurysms involves procedures that either spare or sacrifice the parent vessel. Advancements in flow diverters and stents have allowed for treatments that spare the parent vessels [1]. Initially, these reconstructive methods were considered to be a safer alternative to deconstructive techniques that sacrifice the parent vessel [2], but many groups have now reported higher delayed complications consisting of intracranial hemorrhage, stent thrombosis, and aneurysm rupture [3-5]. For giant, fusiform, or blister aneurysms as well as lesions with difficult access via conventional means, preservation 
of the parent vessel may not be viable and deconstructive techniques such as parent artery occlusion (PAO) may remain the preferable therapy in these scenarios $[1,6]$. Platinum coils [7], detachable balloons [8], and Amplatzer vascular plugs [9-12] have been effectively employed for the goal of achieving PAO.

Earlier literature has accounted for a few series of patients with giant aneurysms [13-15] and a number of smaller series of intracranial aneurysms [16-22] treated with PAO. However, to the authors' knowledge, limited studies [23] to date have directly compared PAO treatment outcomes based on the location of PAO. The purpose of our study was to evaluate the safety and efficacy planned PAO according to its anatomical locations.

\section{Materials and Methods}

We retrospectively reviewed the records of patients with endovascular treatments at our institution since 1999 to 2017. Only those patients with pre-operative planned intentional PAO were included in the final analysis. Patients who experienced inadvertent unplanned PAO during treatment were not included in this study. Additional information was collected regarding demographics, clinical presentations, aneurysmal characteristics, follow-up and complications. Our institutional research ethics board approved this study and informed consent was waived.
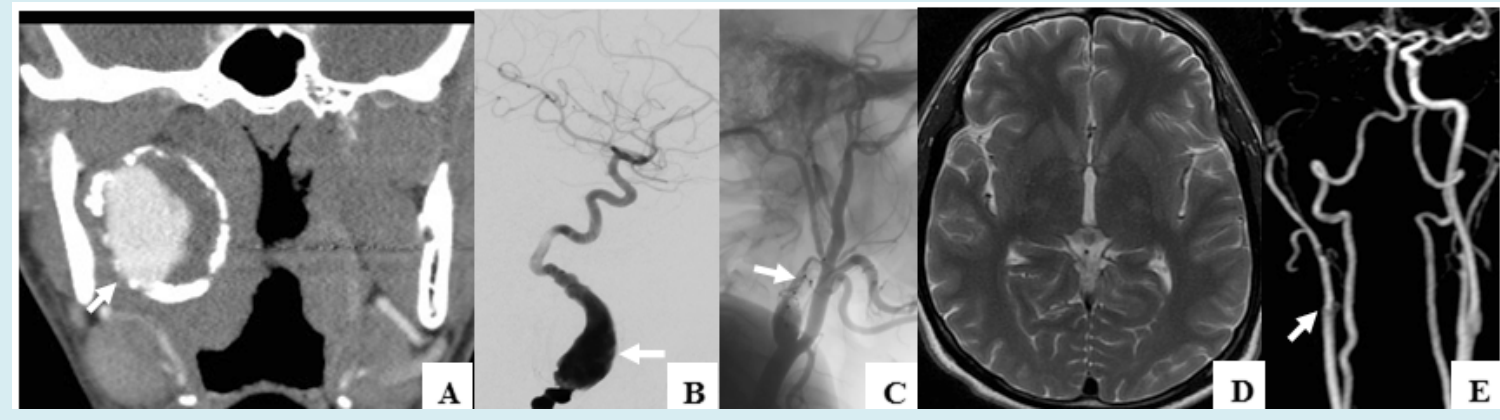

Figure 1: Young patient with large partially thrombosed and calcified, likely chronic dissecting, aneurysm of the right cervical ICA seen on CTA (A); DSA done during PAO procedure shows the large fusiform aneurysm (B) treated with PAO using Amplatzer plugs (arrows) in the proximal ICA (C); MRI of brain on follow-up did not reveal any intracranial ischemic lesion (D) and continued occlusion of the aneurysm (E).
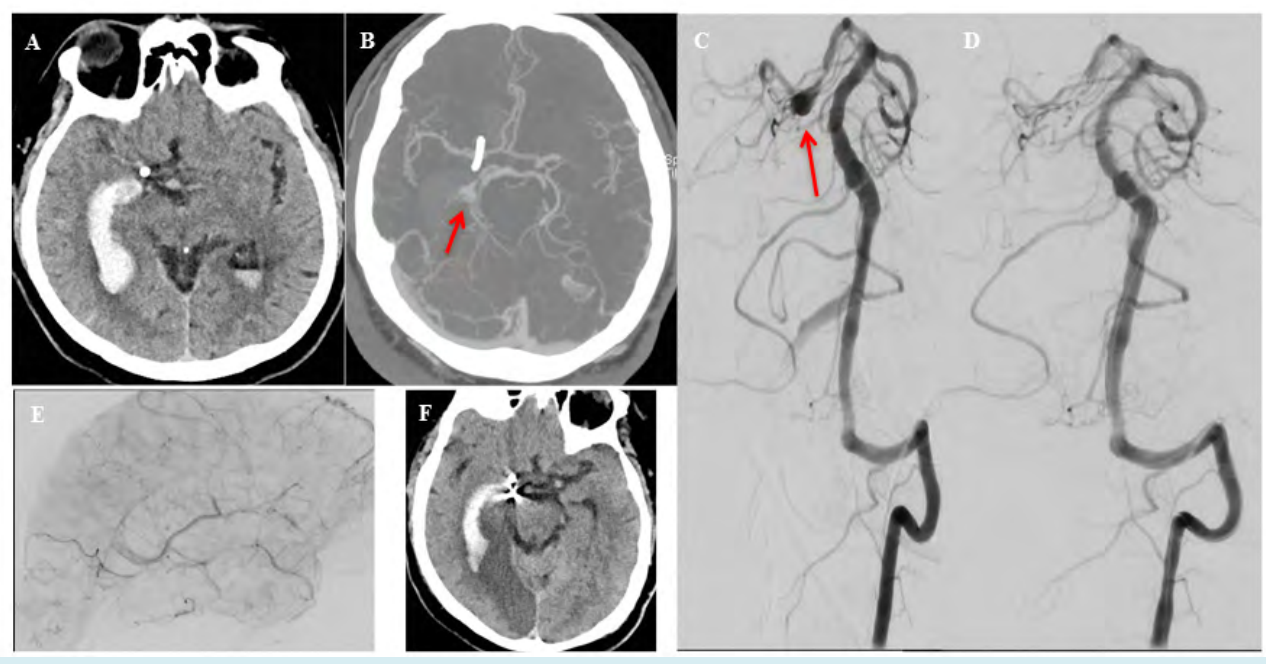

Figure 2: Patient presented with sudden severe headache with CT scan of head showing intraventricular hemorrhage (A) and CT angiogram showing a fusiform aneurysm (arrow), likely dissecting in nature, of the right PCA (B); DSA during the PAO procedure re-demonstrates the PCA aneurysm (arrow, C). Post-interventional DSA shows the aneurysm was occluded completely with no residual filling (D) and delayed filling of the distal PCA via collaterals (E); Post-interventional CT, after 2 days, shows infarct in the right PCA territory (F). 


\section{Clinical Radiology \& Imaging Journal}

Findings were presented according to PAO performed intra-or extra-cranially. Extracranial PAO included those on the internal carotid artery (ICA) (Figure 1) and the extracranial vertebral arteries. Intracranial PAO included those on the anterior cerebral artery (ACA), middle cerebral artery (MCA), posterior cerebral artery (PCA) (Figure 2) and intracranial branches of verbetro-basilar arteries. Information was also collected on balloon test occlusion (BTO).

\section{Statistics}

Simple statistical methods were used to calculate mean, standard deviations and proportions. Odds ratio (OR) was calculated to assess the outcomes of extra- and intra-cranial PAO. Logistic regression was used to control for confounders like age and sex of the patients. A p-value $<0.05$ was considered statistically significant.

\section{Results}

A retrospective review of endovascular treatment of
1000 endovascular treatments from 1999 to 2017 yielded 32 patients (19 women; 13 men) who underwent PAO at our institution. Demographics along with clinical details, location and technical details of PAO are summarized in Table 1. The mean age of the patients was $53 \pm 13.2$ (range 20 to 82 ) years old. Indications for PAO included aneurysm $(n=23)$, dissection $(n=4)$, involvement by tumor $(n=3)$ and acute hemorrhage $(\mathrm{n}=2)$. All patients showed angiographic occlusion on imaging conducted immediately post-PAO. Of the 32 patients, 17 had additional imaging available, with follow-up period ranging from 8 days to 7 years after date of procedure. None of these patients showed recurrence of the aneurysms demonstrating $100 \%$ efficacy of the PAO. BTO was performed only in 11 patients, all of which were for extracranial PAO $52.38 \%$ of 21 patients with extracranial $\mathrm{PAO}$ ). No neurological complications were seen in any of the patients where PAO were performed after BTO. When BTO was not performed, neurological complications were seen in 1 of $10(10 \%)$ extracranial PAO.

\begin{tabular}{|c|c|}
\hline Parameters & $\begin{array}{l}\text { All Subjects } \\
(n=32)\end{array}$ \\
\hline \multicolumn{2}{|l|}{ Sex, n (\%) } \\
\hline Men & $13(40.6 \%)$ \\
\hline Women & $19(59.4 \%)$ \\
\hline \multicolumn{2}{|l|}{ Age, years } \\
\hline Mean \pm SD & $53 \pm 13.4$ \\
\hline Range & $20-82$ \\
\hline \multicolumn{2}{|l|}{ Presentation } \\
\hline SAH & $15(46.9)$ \\
\hline Incidental & $4(12.5 \%)$ \\
\hline $\begin{array}{l}\text { Other (cranial neuropathy, hemiparesis, sudden headache with no } \\
\text { hemorrhage) }\end{array}$ & $13(40.6 \%)$ \\
\hline \multicolumn{2}{|l|}{ Aneurysmal Location } \\
\hline Vertebral & $8(25.0 \%)$ \\
\hline PICA & $3(9.4 \%)$ \\
\hline ICA & $10(31.3 \%)$ \\
\hline CCA & $3(9.4 \%)$ \\
\hline MCA & $3(9.4 \%)$ \\
\hline PCA & $5(15.6 \%)$ \\
\hline \multicolumn{2}{|l|}{ Aneurysmal Type } \\
\hline Dissecting & $8(25.0 \%)$ \\
\hline Fusiform & $2(6.3 \%)$ \\
\hline Giant & $1(3.1 \%)$ \\
\hline
\end{tabular}




\section{Clinical Radiology \& Imaging Journal}

\begin{tabular}{|c|c|}
\hline Other & $21(65.6 \%)$ \\
\hline Technique & \\
\hline Coil & $21(65.6 \%)$ \\
\hline Balloon & $5(15.6 \%)$ \\
\hline Amplatzer Plugs & $3(9.4 \%)$ \\
\hline Balloon \& Coil & $1(3.1 \%)$ \\
\hline Amplatzer Plugs \& Coil & $2(6.3 \%)$ \\
\hline
\end{tabular}

Note: n: number of subjects; SD: standard deviation; SAH: subarachnoid hemorrhage; PICA: posterior inferior cerebellar artery; ICA: internal carotid artery; CCA: common carotid artery; MCA: middle cerebral artery; PCA: posterior cerebral artery.

Table 1: Patient demographics and aneurysm characteristics.

The perioperative complications of permanent neurological deficits were seen in 5 patients $(15.6 \%)$ secondary to infarction in the corresponding arterial territory confirmed on follow-up imaging. One additional patient had intraoperative, non-occlusive vertebral artery dissection with no postoperative clinical consequences. The perioperative permanent neurological deficits were seen in $36.36 \%(4 / 11)$ intracranial PAO and in $4.76 \%(1 / 21)$ of extracranial PAO (OR: 11.43; 95\% confidence interval (CI): 0.83-158.07; $\mathrm{p}=0.02$ ) (Figure 3). The perioperative complications were higher in posterior circulation PAO (3 out of 16; 18.75\%) compared to anterior circulation (2 out of 16; 12.50\%) (OR: 1.61; 95\% CI: 0.22-11.70; $\mathrm{p}=0.63$ ). The permanent neurological deficits for intracranial PAO continued to be higher compared to extracranial PAO (OR: 2.89; 95\% CI: 0.26-5.52; $\mathrm{p}=0.031$ ), when controlled for the age, sex of the patient, and location of aneurysms on posterior versus anterior circulation. Another important confounder for perioperative complications was BTO, which could not be controlled as BTO was not performed in any patients with intracranial PAO.

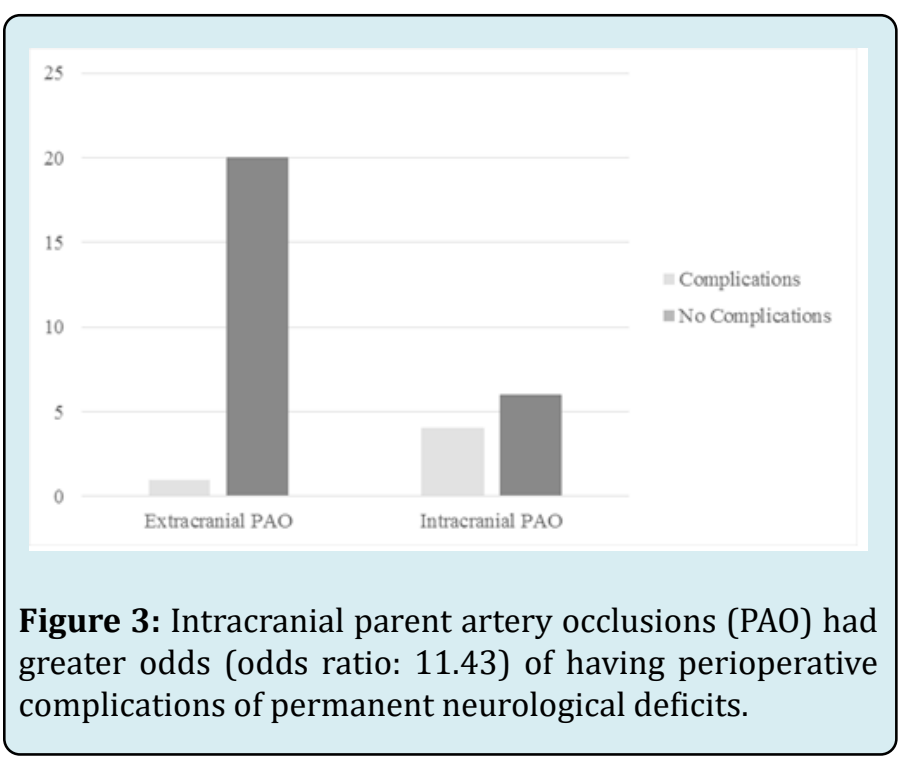

\section{Discussion}

Our small study with short term follow-up confirmed very high efficacy of PAO. This was similar to what was shown in other studies $[14,16-18,23,24]$. The neurological complication rates tend to be higher when PAO was intracranial compared to extracranial (OR: 11.43; $\mathrm{p}=0.02$ ). While this could be obvious, intracranial PAO continued to be an independent predictor of perioperative neurological complication when controlled for age, sex and location of PAO on posterior versus anterior circulation. The lower odds for adverse neurological outcomes for extracranial PAO are likely related to multiple collateral circulation pathways at the level of the circle of Willis (CoW), which aids in keeping the circulation intact. On the other hand, in intracranial PAO, many of these arteries are end arteries with no collaterals [24-26]. Another possibility for the higher complication rates could be a higher technical complications in intracranial PAO versus extracranial PAO. However, this was not found to be higher for intracranial PAO in our study. These findings may help the discussion with the patients and their family before PAO based on the location of PAO.

On contrary, Cui L, et al. reported successful treatment of intracranial aneurysms distal to the CoW with PAO [17]. In their small series of 12 patients; there were 7 PCA, 2 AICA, 1 PICA, and 2 MCA aneurysms. At follow-up, angiograms showed that aneurysms were completely obliterated in all patients with no reports of neurological sequelea [17]. Authors suggested that aneurysms located on the PCA and MCA may be amendable to treatment by PAO given the sufficient collateral supply provided by their distal territories [17,27-30]. Nevertheless, authors proceeded to stress the importance of good candidate selection and appropriate techniques for successful PAO, highlighting the pertinence of preoperative assessment of patients' medical history and symptoms, the anatomy, and the quality of cerebral circulation [17].

We noticed higher perioperative complications rates 


\section{Clinical Radiology \& Imaging Journal}

associated with posterior (18.75\%) versus anterior (12.50\%) circulation PAO, but was not statistically significant $(\mathrm{p}=0.63)$. An earlier study reported higher ischemic complication rate in the posterior circulation $(14.9 \%)$ than the anterior $(10.2 \%)$ circulation [23]. They suggested careful evaluation for tolerance to PAO as well as recommended anti-platelet therapy before PAO and systemic heparinization during PAO for reduction of ischemic events secondary to distal embolism [23].

An invaluable tool for planning PAO and predicting cerebrovascular tolerance includes the BTO, whereby its successful tolerance has been correlated with greater chances of uncomplicated PAO [31]. In our study, 52.38\% of 21 extracranial PAO underwent BTO and none of them suffered neurological complications. In the remaining patients with extracranial PAO, who did not undergo prior BTO, the perioperative neurological complications were observed in $10 \%$ (1 out of 10 ). BTO was not performed in any of our 11 patients with intracranial PAO, where the neurological complications were seen in $36.36 \%$. Although our numbers are small, this may raise discussion about the role of BTO in planning of PAO. The BTO in intracranial circulation is not very well defined and not usually done. In a review of carotid artery occlusion, up to $26 \%$ intraoperative complications and $12 \%$ mortality have been reported. Even in those with successful BTO, a 13\% complications and 3\% mortality have been reported [32].

\section{Limitations}

This was a retrospective study with all the inherent biases of this study type and design. Clinical outcome were assessed by the treating neurointerventionalist rather than by an independent expert. Additional patient and follow-up information were not available. We collected information for patients from as far back as 1999, during which, the advances in technologies and techniques for treatment of these aneurysms could be compared. Notably, all the complications recorded in this study occurred within the past 10 years and the difference did not appear attributable to a specific operator, type of occlusion device, and/or a year of treatment. A major limitation was that we could not control for the effect of successful BTO on the rate of neurological complication in intracranial PAO as no patients with intracranial PAO underwent BTO and no patients had unsuccessful BTO in our series. BTO was performed only for extracranial PAO in our study. This needs to be studied further in larger study.

\section{Conclusion}

Efficacy of PAO in our small study with short term follow up was $100 \%$. Intracranial PAO was an independent predictor for associated neurological morbidity compared to extracranial PAO. Although our numbers were small, BTO appeared to help avoid neurological complications as no patients with successful BTO had any neurological complications.

\section{References}

1. Robertson F, Platts A (2013) Parent Artery Sacrifice. Interventional Neuroradiology, pp: 85-97.

2. Shankar JJ, Tampieri D, Iancu D, Cortes M, Agid R, et al. (2016) SILK flow diverter for complex intracranial aneurysms: a Canadian registry. J Neurointerv Surg 8(3): 273-278.

3. Chow M, McDougall C, O'Kelly C, Ashforth R, Johnson E, et al. (2012) Delayed spontaneous rupture of a posterior inferior cerebellar artery aneurysm following treatment with flow diversion: a clinicopathologic study. Am J Neuroradiol 33(4): 46-51.

4. Klisch J, Turk A, Turner R, Woo HH, Fiorella D (2011) Very late thrombosis of flow-diverting constructs after the treatment of large fusiform posterior circulation aneurysms. Am J Neuroradiol 32(4): 627-632.

5. Lubicz B, Collignon L, Raphaeli G, Pruvo JP, Bruneau M, et al. (2010) Flow-diverter stent for the endovascular treatment of intracranial aneurysms: a prospective study in 29 patients with 34 aneurysms. Stroke 41(10): 2247-2253.

6. Leibowitz R, Do HM, Marcellus ML, Chang SD, Steinberg GK, et al. (2003) Parent vessel occlusion for vertebrobasilar fusiform and dissecting aneurysms. Am J Neuroradiol 24(5): 902-907.

7. Labeyrie MA, Lenck S, Bresson D, Desilles JP, Bisdorff A, et al. (2015) Parent artery occlusion in large, giant, or fusiform aneurysms of the carotid siphon: clinical and imaging results. Am J Neuroradiol 36(1): 140-145.

8. van der Schaaf IC, Brilstra EH, Buskens E, Rinkel GJ (2002) Endovascular treatment of aneurysms in the cavernous sinus: a systematic review on balloon occlusion of the parent vessel and embolization with coils. Stroke 33(1): 313-318.

9. Lee W, Shin YS, Kim KH, Kim YB, Hong CK, et al. (2016) Preliminary Experience with Vascular Plugs for Parent Artery Occlusion of the Carotid or Vertebral Arteries. J Cerebrovasc Endovasc Neurosurg 18(3) :208-214.

10. Ross IB, Buciuc R (2007) The vascular plug: A new device for parent artery occlusion. AJNR 28(2): 385-386. 


\section{Clinical Radiology \& Imaging Journal}

11. Banfield JC, Shankar JJ (2016) Amplatzer vascular plug for rapid vessel occlusion in interventional neuroradiology. Interv Neuroradiol 22(1): 116-121.

12. Shankar JJ, Maloney WJ, Vandorpe R (2011) Amplatzer vascular plug for occlusion of parent artery in carotid blowout with active extravasation. Interv Neuroradiol 17(2): 224-227.

13. Drake CG, Peerless SJ, Ferguson GG (1994) Hunterian proximal arterial occlusion for giant aneurysms of the carotid circulation. J Neurosurg 81(5): 656-665.

14. Clarencon F, Bonneville F, Boch AL, Lejean L, Biondi A (2011) Parent artery occlusion is not obsolete in giant aneurysms of the ICA. Experience with very-long-term follow-up. Neuroradiology 53(12): 973-982.

15. Kai Y, Hamada J, Morioka M, Yano S, Mizuno T, et al. (2007) Treatment strategy for giant aneurysms in the cavernous portion of the internal carotid artery. Surg Neurol 67(2): 148-155.

16. Andreou A, Ioannidis I, Mitsos A (2007) Endovascular treatment of peripheral intracranial aneurysms. Am J Neuroradiol 28(2): 355-361.

17. Cui L, Peng Q Ha W, Zhou D, Xu Y (2009) Parent artery occlusion for intracranial aneurysms. Interv Neuroradiol 15(3): 309-315.

18. Eckard DA, O'Boynick PL, McPherson CM, Eckard VR, Han P, et al. (2000) Coil occlusion of the parent artery for treatment of symptomatic peripheral intracranial aneurysms. Am J Neuroradiol 21(1): 137-142.

19. Ganesh KN, Ladner TR, Kahn IS, Zuckerman SL, Baker CB, et al. (2017) Parent vessel occlusion for treatment of cerebral aneurysms: Is there still an indication? A series of 17 patients. J Neurol Sci 372: 250-255.

20. Khayata MH,Aymard A, Casasco A, Herbreteau D, Woimant F, et al. (1993) Selective endovascular techniques in the treatment of cerebral mycotic aneurysms. Report of three cases. J Neurosurg 78(4): 661-665.

21. Matouk CC, Kaderali Z, TerBrugge KG, Willinsky RA (2012) Long-term clinical and imaging follow-up of complex intracranial aneurysms treated by endovascular parent vessel occlusion. Am J Neuroradiol 33(10): 19911997.

22. Teitelbaum GP, Dowd CF, Larsen DW, McDougall CG, Halbach VV, et al. (1995) Endovascular management of biopsy-related posterior inferior cerebellar artery pseudoaneurysm. Surg Neurol 43(4): 357-359.
23. Ishii A, Miyamoto S, Ito $\mathrm{Y}$, Fujinaka T, Sakai C, et al. (2014) Parent Artery Occlusion for Unruptured Cerebral Aneurysms: The Japanese Registry of Neuroendovascular Therapy (JR-NET) 1 and 2. Neurol Med Chir 54(2): 9197.

24. Fang YB, Lin A, Kostynskyy A, Agid R, Tymianski M, et al. (2018) Endovascular treatment of intracranial vertebrobasilar artery dissecting aneurysms: Parent artery occlusion versus flow diverter. Eur J Radiol 99: 68-75.

25. Liebeskind DS (2003) Collateral circulation. Stroke 34(4): 2279-2284.

26. Silvestrini M, Vernieri F, Troisi E, Passarelli F, Matteis M, et al. (1999) Cerebrovascular reactivity in carotid artery occlusion: possible implications for surgical management of selected groups of patients. Acta Neurol Scand 99(3): 187-191.

27. Arat A, Islak C, Saatci I, Kocer N, Cekirge S (2002) Endovascular parent artery occlusion in large-giant or fusiform distal posterior cerebral artery aneurysms. Neuroradiology 44(8): 700-705.

28. Ciceri EF, Klucznik RP, Grossman RG, Rose JE, Mawad ME (2001) Aneurysms of the posterior cerebral artery: classification and endovascular treatment. AJNR 22(1): 27-34.

29. Hallacq P, Piotin M, MoretJ (2002) Endovascularocclusion of the posterior cerebral artery for the treatment of $\mathrm{p} 2$ segment aneurysms: retrospective review of a 10-year series. AJNR 23(7): 1128-1136.

30. van RooijWJ, Sluzewski M, Beute GN (2006) Endovascular treatment of posterior cerebral artery aneurysms. AJNR 27(2): 300-305.

31. Lesley WS, Rangaswamy R (2009) Balloon test occlusion and endosurgical parent artery sacrifice for the evaluation and management of complex intracranial aneurysmal disease. J Neurointerv Surg 1(2): 112-120.

32. Linskey ME, Jungreis CA, Yonas H, Hirsch WL Jr, Sekhar LN, et al. (1994) Stroke risk after abrupt internal carotid artery sacrifice: accuracy of preoperative assessment with balloon test occlusion and stable xenon-enhanced CT. AJNR 15(5): 829-843. 